\title{
Disruption of Primary Motor Cortex before Learning Impairs Memory of Movement Dynamics
}

\author{
Andrew G. Richardson, ${ }^{1}$ Simon A. Overduin, ${ }^{2}$ Antoni Valero-Cabré, ${ }^{3,4,6}$ Camillo Padoa-Schioppa, ${ }^{5}$ \\ Alvaro Pascual-Leone, ${ }^{6}$ Emilio Bizzi, ${ }^{2}$ and Daniel Z. Press ${ }^{6}$ \\ ${ }^{1}$ Division of Health Sciences and Technology, Massachusetts Institute of Technology and Harvard Medical School, Cambridge, Massachusetts 02142, \\ ${ }^{2}$ Department of Brain and Cognitive Sciences and McGovern Institute for Brain Research, Massachusetts Institute of Technology, Cambridge, \\ Massachusetts 02139, ${ }^{3}$ Department of Anatomy and Neurobiology, Boston University School of Medicine, Boston, Massachusetts 02118, ${ }^{4}$ Department of \\ Neurology, Fondation Ophtalmologique Rothschild, 75019 Paris, France, ${ }^{5}$ Department of Neurobiology, Harvard Medical School, Boston, Massachusetts \\ 02115, and ${ }^{6}$ Department of Neurology, Beth Israel Deaconess Medical Center and Harvard Medical School, Boston, Massachusetts 02215
}

\begin{abstract}
Although multiple lines of evidence implicate the primary motor cortex (M1) in motor learning, the precise role of M1 in the adaptation to novel movement dynamics and in the subsequent consolidation of a memory of those dynamics remains unclear. Here we used repetitive transcranial magnetic stimulation (rTMS) to dissociate the contribution of M1 to these distinct aspects of motor learning. Subjects performed reaching movements in velocity-dependent force fields over three epochs: a null-field baseline epoch, a clockwisefield learning epoch (15 min after the baseline epoch), and a clockwise-field retest epoch ( $24 \mathrm{~h}$ after the learning epoch). Half of the subjects received $15 \mathrm{~min}$ of $1 \mathrm{~Hz}$ rTMS to M1 between the baseline and learning epochs. Subjects given rTMS performed identically to control subjects during the learning epoch. However, control subjects performed with significantly less error than rTMS subjects in the retest epoch on the following day. These results suggest that M1 is not critical to the network supporting motor adaptation per se but that, within this network, M1 may be important for initiating the development of long-term motor memories.
\end{abstract}

Key words: human; motor cortex; learning; memory; rTMS; psychophysics

\section{Introduction}

The human motor system generally uses acquired knowledge of the mechanical properties of both the arm and the environment in the control of reaching behaviors (Shadmehr and MussaIvaldi, 1994; Sainburg et al., 1999). This allows the system to attenuate expected disturbances in an anticipatory manner and improve motor performance. Adapting to a novel dynamic environment involves learning new mechanical properties. Memories of specific environments may over time become resistant to interference from new learning, in a process referred to as motor memory consolidation (Brashers-Krug et al., 1996; Shadmehr and Brashers-Krug, 1997).

Several studies have explored the neural structures underlying adaptation to novel mechanical contexts using functional imaging, transcranial magnetic stimulation (TMS), and neurological populations. A network including the dorsolateral prefrontal cortex, posterior parietal cortex, striatum, and cerebellum is engaged specifically in motor adaptation and not just motor execution (Shadmehr and Holcomb, 1997; Krebs et al., 1998; Nezafat et al., 2001; Della-Maggiore et al., 2004; Maschke et al., 2004; Smith and Shadmehr, 2005). This network may provide both cognitive

Received March 16, 2006; revised 0ct. 25, 2006; accepted 0ct. 26, 2006.

This work was supported by National Institutes of Health Grant K23-MH65434 (D.Z.P.)

Correspondence should be addressed to Dr. Daniel Z. Press, Beth Israel Deaconess Medical Center and Harvard Medical School, 330 Brookline Avenue, Boston, MA 02215. E-mail: dpress@bidmc.harvard.edu. DOI:10.1523/JNEUROSCI.1139-06.2006

Copyright $\odot 2006$ Society for Neuroscience $\quad$ 0270-6474/06/2612466-05\$15.00/0 responses to movement error (Malfait and Ostry, 2004) and error-driven acquisition of an internal model of the movement dynamics (Kawato, 1999). As the memory of the movement dynamics consolidates, activity partially shifts to a network including premotor cortex, posterior parietal cortex, and cerebellar cortex, which may store the internal model (Shadmehr and Holcomb, 1997; Krebs et al., 1998; Nezafat et al., 2001).

Human primary motor cortex (M1) is involved in several types of motor skill learning (Pascual-Leone et al., 1994; Karni et al., 1995; Ghilardi et al., 2000; Sanes and Donoghue, 2000) but does not appear to be differentially activated when adapting to new movement dynamics compared with baseline execution (Shadmehr and Holcomb, 1997; Krebs et al., 1998). Single-unit recordings in monkeys, however, suggest that involvement of M1 in motor adaptation may not be apparent in more global measures of activity. In particular, distributed subsets of M1 neurons appear to support a memory trace of novel movement dynamics, whereas the population as a whole reflects only the task execution (Li et al., 2001).

The present study aimed to more directly assess the role of M1 in human adaptation to novel dynamical environments. We used low-frequency, repetitive TMS (rTMS) to interfere with M1 function while subjects performed reaching movements in a velocity-dependent force field. Applying rTMS to M1 before the first exposure to the force field, we tested subjects' ability to adapt to the novel dynamics as well as their ability to recall these dynamics $24 \mathrm{~h}$ later. We found that M1 disruption did not affect 

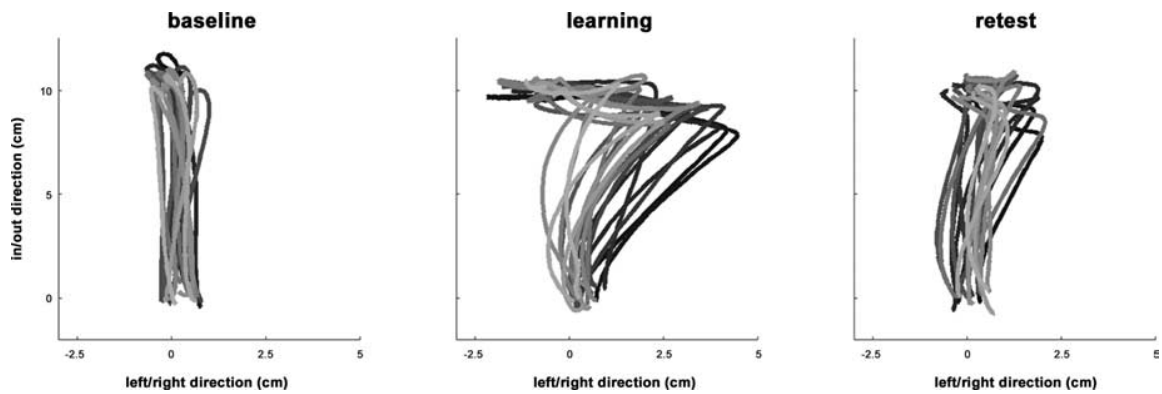

Figure 1. Subjects adapted to a velocity-dependent force field introduced in the learning epoch and repeated at retest $24 \mathrm{~h}$ later. The sample trajectories include all those performed by one control subject in the forward direction of reach, from among the 150 baseline trials and the first 150 trials performed in the two clockwise-field epochs. The temporal order of the trajectories within each epoch is given by the transition from black (early trials) to gray (late trials).

initial adaptation but did hinder next-day performance relative to controls. These results suggest that a network including M1 may be critical to the early stages of motor memory development.

\section{Materials and Methods}

Paradigm. Sixteen right-handed subjects (mean, 25 years old; six males) participated in the experiment. Participants were screened for history of seizures, familial epilepsy, and other TMS contraindications. Ethical approval was obtained through the Massachusetts Institute of Technology Committee on the Use of Humans as Experimental Subjects. The subjects were randomly assigned to two experimental groups ("control" and "rTMS").

Subjects were instructed to hold onto a robotic manipulandum with their right hand and make reaching movements in the horizontal plane to targets presented on a vertically oriented monitor (for details, see Shadmehr and Mussa-Ivaldi, 1994). Targets included four peripheral squares spaced around a central square at a distance of $10 \mathrm{~cm}$, such that the movements from either central to peripheral or peripheral to central resulted in eight movement directions, uniformly spanning $360^{\circ}$. The peripheral squares were located at $0,45,90$, and $135^{\circ}$, according to the direction labels in Figure 2C. Subjects were given $0.50 \pm 0.05$ s to complete each movement. Trials completed in the specified time were indicated to the subject by a brief sound. Trials completed too quickly or too slowly were indicated to the subject by a transition in the target color from white to red or blue, respectively.

Participants performed reaching movements to a pseudorandom sequence of targets in three different epochs, denoted as "baseline" (two subepochs of $253 \pm 8$ trials and $152 \pm 4$ trials separated by $15 \mathrm{~min}$ ), "learning" (400 \pm 0 trials), and "retest" (403 \pm 10 trials; mean \pm SD). The number of trials in each (sub)epoch for the control group and rTMS group did not significantly differ. The duration of the interval between the baseline and learning epochs was $\sim 15 \mathrm{~min}$. The learning and retest epochs were separated by $24 \mathrm{~h}$.

All subjects experienced a null $\left(0 \mathrm{~N} \cdot \mathrm{s} \cdot \mathrm{m}^{-1}\right)$ force field during the baseline epoch and a velocity-dependent clockwise field generated by the robotic manipulandum in the learning and retest epochs. The curl forces, of magnitude $15 \mathrm{~N} \cdot \mathrm{s} \cdot \mathrm{m}^{-1}$, were calculated on-line as $f=\mathbf{B} \dot{x}$, where

$$
\mathbf{B}=\left[\begin{array}{cc}
0 & 15 \\
-15 & 0
\end{array}\right]
$$

and $\dot{x}$ was the movement velocity.

TMS was delivered using a Magstim (Whitland, Wales, UK) Super Rapid stimulator. Between the two subepochs of the baseline epoch, the location and threshold for stimulation were determined in the rTMS subjects. This involved using single pulses of TMS over the left motor cortex to determine (1) the scalp location capable of reliably and maximally inducing visible contractions in the right biceps brachii muscle, and (2) the intensity threshold necessary to reliably elicit a motor-evoked potential in the resting right biceps muscle following standard criteria (at least $50 \mu \mathrm{V}$, present in 5 of 10 consecutive attempts). The intensity level of the rTMS used in the experiment was then calculated to be $90 \%$ of the resting biceps motor threshold level, an intensity known to induce long-lasting depression of motor cortex excitability (Gangitano et al., 2002; Romero et al., 2002). Between the baseline and learning epochs, rTMS pulses were applied at a frequency of $1 \mathrm{~Hz}$ for $15 \mathrm{~min}$ (i.e., 900 pulses) using a hand-held figure-of-eight coil (double $70 \mathrm{~mm}$; Magstim), positioned tangentially relative to the scalp in a $45^{\circ}$ posterior-toanterior and lateral-to-medial orientation, at the same location found to optimally evoke biceps activity during localization.

We did not use sham stimulation in control subjects because the rTMS was delivered "offline," while the subject was at rest before force field exposure. Thus, it was unlikely that any nonspecific attentional or behavioral effects of the TMS were present during the subsequent learning epoch (Robertson et al., 2003). In support of this claim, we found that rTMS and control subjects' performance in the learning epoch (i.e., just after rTMS was applied) did not significantly differ, arguing that rTMS had no immediate, specific or nonspecific effect on motor performance (see Fig. $2 \mathrm{~A}$ and Results).

Analysis. We quantified performance on each trial as the signed peak perpendicular deviation relative to a straight line connecting the beginning and end positions of the trial (cf. Shadmehr and Moussavi, 2000). This performance measure was normalized in each epoch and for each group by subtracting the average baseline epoch performance of the group. The normalization removed intergroup differences in baseline task performance to isolate learning-specific performance changes. Note that without normalization the pattern of statistically significant results was not altered. Trials in which the subject failed to reach the target within a $0.50 \pm 0.25 \mathrm{~s}$ time window were excluded from the analysis. Trials were binned by 16 trials in each epoch and group. Statistical results were based on the within-bin-averaged perpendicular deviation. Not all subjects were given exactly 400 trials to complete in each of the learning and retest epochs (see above); hence, we only used the first 24 (rather than 25) 16-trial bins in each of these epochs for the statistical tests. Main and interaction effects of rTMS, time (either individual or grouped time bins; see Results), and movement direction were assessed using repeatedmeasures ANOVAs. All significant effects (at the $p<0.05$ level) are reported.

To assess the robustness of our results, we repeated the analysis using two other measures of performance: signed deviation angle (the angle between the lines connecting beginning and end positions and beginning and maximum speed positions) (cf. Della-Maggiore et al., 2004) and signed deviation area (the area between the hand trajectory and the line connecting beginning and end positions). The results using each of these measures were very similar; for brevity, we report only the analysis using deviation angle in Results (see Fig. 2B).

\section{Results}

Subjects exhibited a typical pattern of adaptation to the velocitydependent force field (Fig. 1) (Shadmehr and Mussa-Ivaldi, 1994). Reaching trajectories produced in the clockwise force field environment were initially deviated in the clockwise direction. With practice, in both the learning and retest epoch, the trajectories returned to a less-deviated form like that exhibited under null-field conditions.

In the baseline epoch, the performance was stable for both rTMS and control groups (i.e., there was no significant effect of time), and there were no significant differences between the groups (Fig. $2 \mathrm{~A}$, left). After the baseline epoch, the rTMS subjects received 15 min of $1 \mathrm{~Hz}$ rTMS centered over M1, whereas control subjects waited for an equivalent time while seated in the same position. Immediately after this interval, all participants performed a 400-trial learning epoch in a clockwise force field envi- 

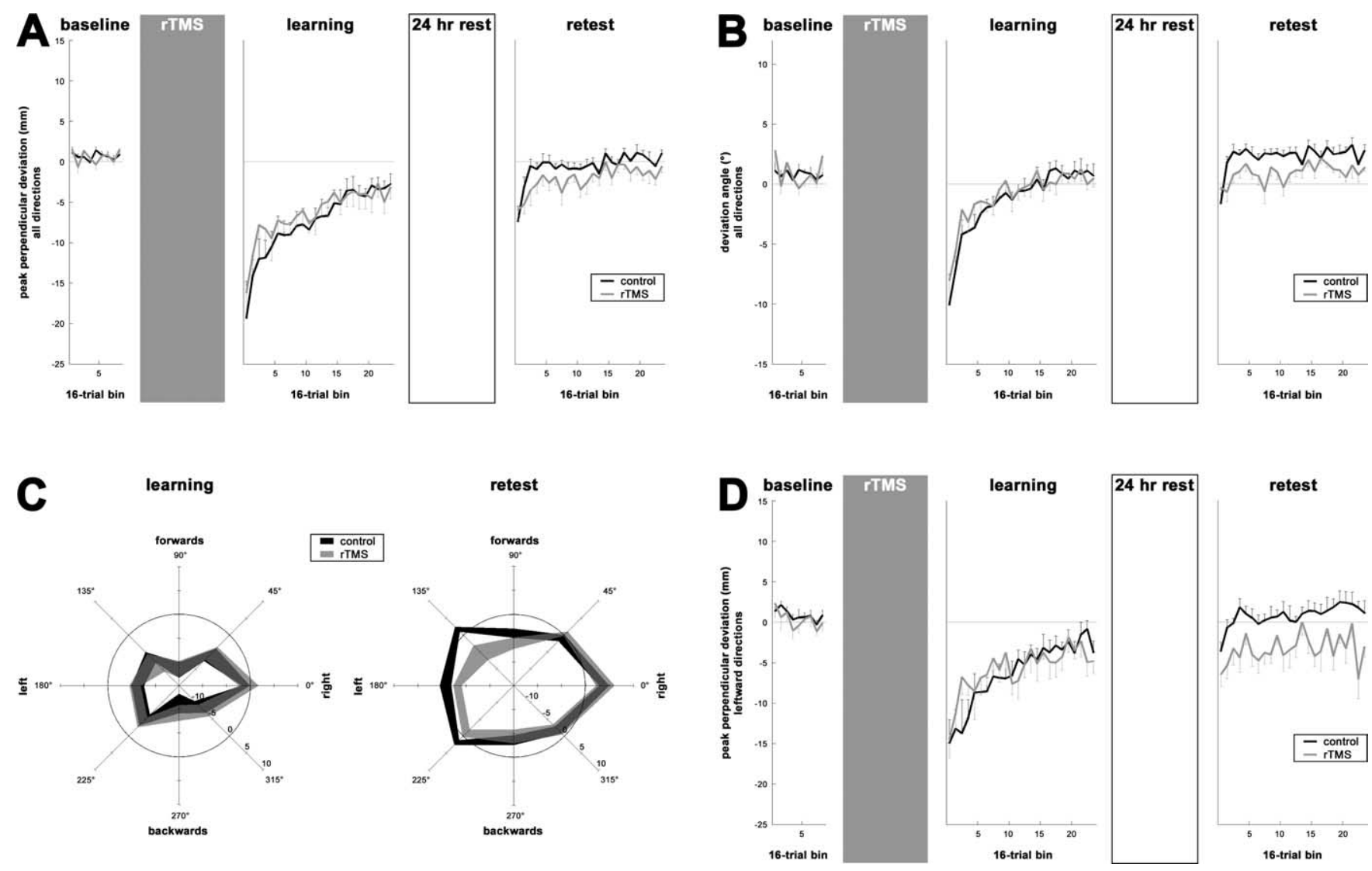

Figure 2. rTMS of M1 spares initial motor performance and force field acquisition but impairs retest performance. $\boldsymbol{A}$, The performance (quantified by the baseline-normalized peak perpendicular deviation) of control (black) and rTMS (gray) groups are shown for baseline, learning, and retest trials (mean \pm SEM of 16-trial bins; bottom error bar for control group and top error bar for rTMS group are removed for clarity). $\boldsymbol{B}$, Same as $\boldsymbol{A}$ but using the baseline-normalized deviation angle as the performance measure. $\boldsymbol{C}$, The average learning and retest performance error for control (black) and rTMS (gray) groups is shown for each movement direction (shaded region indicates \pm 1 SEM around the mean performance over the entire epoch). The performance (shown on the radial axis) is quantified by the peak perpendicular deviation, in which negative errors indicate clockwise deviation, as in $\boldsymbol{A}$. $\boldsymbol{D}$, Same as $\boldsymbol{A}$ but restricted to only leftward-directed $\left(135,180\right.$, and $\left.225^{\circ}\right)$ movements.

ronment generated by the robotic manipulandum. Subjects adapted to the force field (Fig. $2 \mathrm{~A}$, middle), as supported by a significant effect of time on the peak perpendicular deviation $\left(F_{(23,322)}=24.48 ; p<0.0001\right)$. Notably, there was no significant effect of rTMS on subjects' performance.

An effect of rTMS was, however, apparent when the participants returned $24 \mathrm{~h}$ after learning for the retest epoch. Within the 400-trial retest epoch, subjects again adapted to the clockwise force field (Fig. $2 \mathrm{~A}$, right), captured as before by a significant main effect of time $\left(F_{(23,322)}=8.33 ; p<0.0001\right)$. However, in contrast to the learning epoch, there was also a significant main effect of rTMS $\left(F_{(1,14)}=3.15 ; p=0.0482\right)$, with the rTMS group performing with a higher level of error than the control group. This relative difference in error cannot be attributed to a relative difference in movement speed (to which the forces were proportional), because there was no significant main or interaction effect of rTMS on peak speed in the retest (or any other) epoch.

rTMS did not completely interfere with the memory of the novel dynamics experienced in the learning epoch, because both groups performed with significantly less error at the beginning of the retest epoch than they did at the beginning of the learning epoch, although to different degrees. Indeed, in a comparison of the peak perpendicular deviation in the first third of the learning and retest epochs (Fig. $2 A$ ), there were significant effects of both time and its interaction with $\mathrm{rTMS}\left(F_{(1,14)}=100.67, p<0.0001\right.$; and $F_{(1,14)}=6.47, p=0.0089$, respectively). In fact, beyond the first few trials, the initial retest performance error was even less than late-learning epoch error, particularly for the control group. Again, significant time and rTMS $\times$ time effects $\left(F_{(1,14)}=8.36\right.$, $p=0.0040$; and $F_{(1,14)}=3.54, p=0.0383$, respectively) were evident in a comparison of the last third of the learning epoch with the first third of the retest epoch (Fig. 2A).

In addition, the effect of rTMS in the retest epoch was not uniform over all eight movement directions (Fig. $2 \mathrm{C}$, right). A repeated-measures ANOVA, again using the peak perpendicular deviation measure, revealed significant main effects of rTMS $\left(F_{(1,14)}=3.84 ; p=0.0325\right)$ and direction $\left(F_{(7,98)}=13.47 ; p<\right.$ $0.0001)$, as well as a significant rTMS $\times$ direction interaction $\left(F_{(7,98)}=2.71 ; p=0.0292\right)$. In particular, the rTMS group performed with relatively more error only in leftward-directed movements (Fig. $2 C$, right). In contrast, for the learning epoch (Fig. 2C, left), the same repeated-measures ANOVA revealed no significant main or interaction effects of rTMS, only a significant main effect of direction $\left(F_{(7,98)}=25.52 ; p<0.0001\right)$. Thus, the movement direction-specific effect of rTMS was only present at retest, not during initial learning.

All of the statistically significant trends reported for the alldirection analysis were seen when restricting the analysis to just the three leftward-directed $\left(135,180\right.$, and $\left.225^{\circ}\right)$ movements (Fig. $2 D$ ). In particular, during the baseline epoch (Fig. $2 D$, left), there was no effect of time or rTMS on performance. During the learning epoch (Fig. $2 D$, middle), there was only a significant main effect of time on the peak perpendicular deviation $\left(F_{(23,322)}=\right.$ $11.18 ; p<0.0001$ ). During the retest epoch (Fig. $2 D$, right), their 
were significant main effects of both time and rTMS $\left(F_{(22,308)}=\right.$ 2.83, $p=0.0002$; and $F_{(1,14)}=9.69, p=0.0024$, respectively). This analysis again shows that the directionally specific effect of rTMS during retest performance was not also present during initial acquisition of the motor skill.

Finally, the results presented above were robust in that they could be replicated using other performance measures. For example, using deviation angle as the measure of performance (see Materials and Methods), there were again no significant changes in the baseline epoch (Fig. $2 \mathrm{~B}$, left) and only a main effect of time $\left(F_{(23,322)}=25.04 ; p<0.0001\right)$ in the learning epoch (Fig. $2 B$, middle). In the retest epoch (Fig. $2 B$, right), there were significant effects of time and rTMS $\left(F_{(23,322)}=3.94, p<0.0001\right.$; and $F_{(1,14)}=$ $10.57, p=0.0018$, respectively), as reported previously with the peak perpendicular deviation measure. Note that, in the retest epoch, the deviation angle measure indicates that the control group actually overcompensates for the clockwise force field (i.e., has counterclockwise error), whereas the error of the rTMS group is closer to zero (Fig. $2 \mathrm{~B}$, right). It has been shown previously that increasing uninterrupted practice in a force field leads to increasing overcompensation of movement trajectories (Thoroughman and Shadmehr, 2000). Thus, we interpret the greater counterclockwise error as being more adaptive and, consequently, that the rTMS group was impaired relative to the control group, consistent with our previous conclusion.

\section{Discussion}

In this experiment, we studied the role of M1 in the adaptive control of human reaching movements. We used rTMS to disrupt M1 function just before subjects' initial exposure to a velocity-dependent force field. rTMS had no effect on initial performance or adaptation to the force field. However, when subjects performed in the same force field $24 \mathrm{~h}$ later, those who had received rTMS the day before performed worse than those who had not.

The use of a 15 min train of subthreshold $1 \mathrm{~Hz}$ rTMS was specifically chosen to depress M1 excitability for the duration of the learning epoch (Chen et al., 1997; Gangitano et al., 2002; Romero et al., 2002). Nevertheless, we found that rTMS subjects and control subjects performed equally in this epoch. Given that changes in M1 excitability are known to be sufficient to measurably affect behavior (Pascual-Leone et al., 1994), the implication is either that M1 is not involved in initial motor adaptation (Diedrichsen et al., 2005; Paz et al., 2005) or that its involvement can be acutely compensated by the recruitment of other brain areas (Lee et al., 2003). Indeed, although changes in M1 neuronal activity mirror those of muscle activity in force field adaptation (Li et al., 2001), other cortical motor areas show similar activity changes (Padoa-Schioppa et al., 2004; Xiao et al., 2006). The non-necessity of M1 in motor performance and early motor learning has been documented previously in both force field tasks and ballistic finger movement tasks (Muellbacher et al., 2002; Baraduc et al., 2004). It is important to note, however, that other motor cortical and even subcortical areas may also have been influenced by the stimulation, by either transynaptic transmission or direct volume conduction of the magnetic field effects (Chouinard et al., 2003), although the latter should have been quite minimal (Fox et al., 1997).

The novel result in our study was the effect of rTMS on retest performance. Both groups exhibited some memory of the force field in the retest epoch, because initial retest errors were not as large as those of the early learning epoch. However, the rTMS subjects exhibited a relative memory impairment. The memory impairment was not compensated by the return of M1 to normal function $\sim 10$ min after the end of stimulation, once the effects of the stimulation subsided (Romero et al., 2002). This suggests that M1 plays an important role early in motor memory formation, starting specifically at the time of acquisition. This conclusion is substantiated by recent electrophysiological evidence that M1 supports a short-term memory trace of novel movement dynamics (Li et al., 2001).

The relative impairment in performance of the rTMS group in the retest epoch was manifest only in leftward movement directions. This directional selectivity was an unexpected finding, but the cortical representation of arm muscles as well as their activation in force field learning may offer an explanation. The leftward-directional tuning of the impairment is nearly identical to the directionally specific recruitment pattern of the biceps brachii muscle in a clockwise velocity-dependent force field (Thoroughman and Shadmehr, 1999, their Fig. 7). The rTMS protocol used here targeted the M1 representation of the biceps muscle, but selective stimulation of this representation is unlikely given the close proximity of other muscle representations in motor cortex (Schieber, 2001) and the known spatial resolution of TMS (Siebner and Rothwell, 2003). Rather, the similarity in directional tuning of the impairment and the biceps recruitment may be related to the distribution of corticospinal projections to proximal arm motor neuron pools. In humans and other primates, there are significantly more cortico-motoneuronal cells projecting to biceps than triceps motor neurons (Palmer and Ashby, 1992). Furthermore, contrary to traditional clinical assumptions, lesions in human motor cortex lead to a corresponding distribution of proximal arm weakness: elbow flexors are affected to a relatively greater extent than elbow extensors in the paretic limb (Colebatch et al., 1986; Colebatch and Gandevia, 1989; Andrews and Bohannon, 2000). Thus, we suggest that the "virtual lesion" created by rTMS had relatively greater effect on directions of movement that involved the greatest biceps activation. This may have preferentially impaired the memory of the dynamics in these directions, assuming that the process of early memory formation is sufficiently local and not as robust to M1 disruption as motor performance.

In some respects, our results are similar to those of Muellbacher et al. (2002) who found, using a ballistic finger movement task, that rTMS to M1 immediately after learning interfered with retention of the motor skill. They concluded that M1 is involved in early motor memory consolidation, in which consolidation was defined as the stabilization of the memory (i.e., resistance to interference). In our study, we applied rTMS before rather than after learning, but it similarly interfered with the motor memory. However, unlike the Muellbacher et al. (2002) study, the interference did not bring retest performance in the force field back to a naive state. This was true even if one considers only leftwarddirected movements (Fig. 2D). This difference in degree of learning retention after $\mathrm{M} 1$ disruption between a ballistic movement task (as by Muellbacher et al., 2002) and a force field adaptation task (as in our study) was noted explicitly by Baraduc et al. (2004). However, unlike Baraduc et al. (2004), our study identified an important role for M1 in motor memory formation of novel dynamics, one that is not related to memory stabilization.

Indeed, the TMS interference in our study resulted in a pattern of retest performance that more closely resembles a second form of consolidation documented in the procedural memory literature, an off-line skill improvement that occurs between practice sessions (Robertson et al., 2004). In particular, immediately after the first few trials (during which cognitive systems may 
dominate the behavior as subjects readjust to the experimental conditions), the control group showed an improved performance relative to their error level late in the learning epoch. In contrast, the rTMS group showed no such improvement. Therefore, disruption of a network including M1 may selectively impair off-line skill enhancement, as shown in sequence learning tasks (Robertson et al., 2005). Although additional work will be needed to specifically address this issue, our present findings are remarkable for their implication that proper M1 function during adaptation to a novel dynamical environment is important for the full development of a memory of these dynamics.

\section{References}

Andrews AW, Bohannon RW (2000) Distribution of muscle strength impairments following stroke. Clin Rehabil 14:79-87.

Baraduc P, Lang N, Rothwell JC, Wolpert DM (2004) Consolidation of dynamic motor learning is not disrupted by rTMS of primary motor cortex. Curr Biol 14:252-256.

Brashers-Krug T, Shadmehr R, Bizzi E (1996) Consolidation in human motor memory. Nature 382:252-255.

Chen R, Classen J, Gerloff C, Celnik P, Wassermann EM, Hallett M, Cohen LG (1997) Depression of motor cortex excitability by low-frequency transcranial magnetic stimulation. Neurology 48:1398-1403.

Chouinard PA, Van Der Werf YD, Leonard G, Paus T (2003) Modulating neural networks with transcranial magnetic stimulation applied over the dorsal premotor and primary motor cortices. J Neurophysiol 90:1071-1083.

Colebatch JG, Gandevia SC (1989) The distribution of muscular weakness in upper motor neuron lesions affecting the arm. Brain 112:749-763.

Colebatch JG, Gandevia SC, Spira PJ (1986) Voluntary muscle strength in hemiparesis: distribution of weakness at the elbow. J Neurol Neurosurg Psychiatry 49:1019-1024.

Della-Maggiore V, Malfait N, Ostry DJ, Paus T (2004) Stimulation of the posterior parietal cortex interferes with arm trajectory adjustments during the learning of new dynamics. J Neurosci 24:9971-9976.

Diedrichsen J, Hashambhoy Y, Rane T, Shadmehr R (2005) Neural correlates of reach errors. J Neurosci 25:9919-9931.

Fox P, Ingham R, George MS, Mayberg H, Ingham J, Roby J, Martin C, Jerabek P (1997) Imaging human intra-cerebral connectivity by PET during TMS. NeuroReport 8:2787-2791.

Gangitano M, Valero-Cabré A, Tormos JM, Mottaghy FM, Romero JR, Pascual-Leone A (2002) Modulation of input-output curves by low and high frequency repetitive transcranial magnetic stimulation of the motor cortex. Clin Neurophysiol 113:1249-1257.

Ghilardi M, Ghez C, Dhawan V, Moeller J, Mentis M, Nakamura T, Antonini A, Eidelberg D (2000) Patterns of regional brain activation associated with different forms of motor learning. Brain Res 871:127-145.

Karni A, Meyer G, Jezzard P, Adams MM, Turner R, Ungerleider LG (1995) Functional MRI evidence for adult motor cortex plasticity during motor skill learning. Nature 377:155-158.

Kawato M (1999) Internal models for motor control and trajectory planning. Curr Opin Neurobiol 9:718-727.

Krebs HI, Brashers-Krug T, Rauch SL, Savage CR, Hogan N, Rubin RH, Fischman AJ, Alpert NM (1998) Robot-aided functional imaging: application to a motor learning study. Hum Brain Mapp 6:59-72.

Lee L, Siebner HR, Rowe JB, Rizzo V, Rothwell JC, Frackowiak RS, Friston KJ (2003) Acute remapping within the motor system induced by lowfrequency repetitive transcranial magnetic stimulation. J Neurosci 23:5308-5318.

Li CR, Padoa-Schioppa C, Bizzi E (2001) Neuronal correlates of motor per- formance and motor learning in the primary motor cortex of monkeys adapting to an external force field. Neuron 30:593-607.

Malfait N, Ostry DJ (2004) Is interlimb transfer of force-field adaptation a cognitive response to the sudden introduction of load? J Neurosci 24:8084-8089.

Maschke M, Gomez CM, Ebner TJ, Konczak J (2004) Hereditary cerebellar ataxia progressively impairs force adaptation during goal-directed arm movements. J Neurophysiol 91:230-238.

Muellbacher W, Ziemann U, Wissel J, Dang N, Kofler M, Faccini S, Boroojerdi B, Poewe W, Hallett M (2002) Early consolidation in human primary motor cortex. Nature 415:640-644.

Nezafat R, Shadmehr R, Holcomb HH (2001) Long-term adaptation to dynamics of reaching movements: a PET study. Exp Brain Res 140:66-76.

Padoa-Schioppa C, Li CR, Bizzi E (2004) Neuronal activity in the supplementary motor area of monkeys adapting to a new dynamical environment. J Neurophysiol 91:449-473.

Palmer E, Ashby P (1992) Corticospinal projections to upper limb motoneurones in humans. J Physiol (Lond) 448:397-412.

Pascual-Leone A, Grafman J, Hallett M (1994) Modulation of cortical motor output maps during development of implicit and explicit knowledge. Science 263:1287-1289.

Paz R, Natan C, Boraud T, Bergman H, Vaadia E (2005) Emerging patterns of neuronal responses in supplementary and primary motor areas during sensorimotor adaptation. J Neurosci 25:10941-10951.

Robertson EM, Theoret H, Pascual-Leone A (2003) Studies in cognition: the problems solved and created by transcranial magnetic stimulation. J Cogn Neurosci 15:948-960.

Robertson EM, Pascual-Leone A, Miall RC (2004) Current concepts in procedural consolidation. Nat Rev Neurosci 5:1-7.

Robertson EM, Press DZ, Pascual-Leone A (2005) Off-line learning and the primary motor cortex. J Neurosci 25:6372-6378.

Romero JR, Anschel D, Sparing R, Gangitano M, Pascual-Leone A (2002) Subthreshold low frequency repetitive transcranial magnetic stimulation selectively decreases facilitation in the motor cortex. Clin Neurophysiol 113:101-107.

Sainburg RL, Ghez C, Kalakanis D (1999) Intersegmental dynamics are controlled by sequential anticipatory, error correction, and postural mechanisms. J Neurophysiol 81:1045-1056.

Sanes JN, Donoghue JP (2000) Plasticity and primary motor cortex. Annu Rev Neurosci 23:393-415.

Schieber MH (2001) Constraints on somatotopic organization in the primary motor cortex. J Neurophysiol 86:2125-2143.

Shadmehr R, Brashers-Krug T (1997) Functional stages in the formation of human long-term motor memory. J Neurosci 17:409-419.

Shadmehr R, Holcomb HH (1997) Neural correlates of motor memory consolidation. Science 277:821-825.

Shadmehr R, Moussavi ZMK (2000) Spatial generalization from learning dynamics of reaching movements. J Neurosci 20:7807-7815.

Shadmehr R, Mussa-Ivaldi FA (1994) Adaptive representation of dynamics during learning of a motor task. J Neurosci 14:3208-3224.

Siebner HR, Rothwell J (2003) Transcranial magnetic stimulation: new insights into representational cortical plasticity. Exp Brain Res 148:1-16.

Smith MA, Shadmehr R (2005) Intact ability to learn internal models of arm dynamics in Huntington's disease but not cerebellar degeneration. J Neurophysiol 93:2809-2821.

Thoroughman KA, Shadmehr R (1999) Electromyographic correlates of learning an internal model of reaching movements. J Neurosci 19:8573-8588.

Thoroughman KA, Shadmehr R (2000) Learning of action through adaptive combination of motor primitives. Nature 407:742-747.

Xiao J, Padoa-Schioppa C, Bizzi E (2006) Neuronal correlates of movement dynamics in the dorsal and ventral premotor area in the monkey. Exp Brain Res 168:106-119. 